\title{
Pose Estimation of Unresolved Targets using Polarimetric Imaging
}

\author{
Michael Gartley*a, Peter Erbach ${ }^{\mathrm{b}}$, Larry Pezzaniti ${ }^{\mathrm{b}}$ \\ ${ }^{a}$ Digital Imaging and Remote Sensing Laboratory, Rochester Institute of Technology, \\ 54 Lomb Memorial Drive, Rochester, NY 14623-5604 \\ ${ }^{\mathrm{b}}$ Polaris Sensor Technologies, Inc., P.O. Box 8048, Philadelphia PA, 19101;
}

\begin{abstract}
Polarimetric remote sensing has demonstrated utility for improving contrast between man-made targets and complex backgrounds. More specifically, polarimetric signatures of man-made targets can be useful for cueing analysts in widearea search applications. However, taking the target cueing to the next level of identification and tracking may require tasking of other sensing modalities. We present research that aims to understand what pose information might be extracted from the polarimetric signatures of under-resolved targets. Through experimentally collected and simulated imagery, we examine the variation in target signatures with respect pose and the relative sensor position aiming to extend the intelligence value of polarimetric imagery.
\end{abstract}

Keywords: Polarimetry, passive imaging, thermal, pose.

\section{INTRODUCTION}

The utility of polarimetric imaging (PI) systems has been demonstrated by many to improve contrast between man-made targets and natural clutter [1,2]. When the ground-sample-distance (GSD) of the PI system enables multiple pixels on target, the man-made objects highlighted by PI may be identified and characterized [3] based on visual exploitation. However, a more challenging problem exists for targets where only a few to a single pixel on target is possible. In these cases, PI still has the potential for cueing an image analyst to areas of interest, but limits their ability to distinguish the shape of the target and its absolute orientation or pose.

\section{TECHNICAL APPROACH}

For this research, we utilized the Digital Image and Remote Sensing Image Generation (DIRSIG) simulation model to generate spatially oversampled emissive Stoke's imagery of vehicular target's on flat, infinite plane backgrounds. The DIRSIG model is an image generation tool that utilizes a complex computational radiometry sub-system to predict absolute fluxes within a 3D scene description. The model uses [1 x 4] Stokes vector and [4 x 4] Mueller matrix calculus to propagate, reflect, transmit, etc. fluxes within the simulated scene environment. The DIRSIG radiometry engine utilizes a single expression, governing equation across all wavelength regions such that reflected and self-emitted contributions are always included unless explicitly disabled.

The DIRSIG model has a flexible radiometry sub-system for computing radiance values for arbitrary paths within the defined scene. The primary mechanism used to predict images is reverse ray-tracing where rays originate from the imaging detectors and are propagated into the scene. When a ray intersects the scene geometry, the associated radiometry solver is run to compute the surface leaving radiance. DIRSIG has a handful of radiometry solvers used for opaque surfaces and the most flexible is the generic radiometry solver. The generic radiometry solver computes the reflected radiance by sampling the hemisphere above the target. The distribution of these samples is based on the shape and magnitude of the associated BRDF. The nominal hemispherical sampling is cosine projected and has user-defined sampling parameters (e.g. total number of samples, etc.). The incident loads for those samples are determined by tracing higher generation rays which intersect other surfaces and trigger other instances of the radiometry solver. The fidelity of the sampling for higher generation bounces can be decreased using a bounce-dependent decay rate that modifies the sampling parameters. The total number of bounces that are tracked is also user controllable. The incident loads from the

Polarization: Measurement, Analysis, and Remote Sensing IX, edited by David B. Chenault, Dennis H. Goldstein, Proc. of SPIE Vol. 7672, 767204 · C 2010 SPIE · CCC code: 0277-786X/10/\$18 · doi: 10.1117/12.851506 
sampled hemisphere are numerically integrated using the geometry specific reflectance (BRDF) and the solid angle of the sample.

\subsection{Imaging Scenario and Targets}

For this study we have chosen to image a variety of automobiles against an infinite plane background of uniform temperature and emissivity. The automobiles are each attributed with the same material properties, namely a glossy red paint, a rubber material for tires and dark plastic details (such as the soft top of the Miata), a polished metal for hubcaps and metal trim, and finally a simple specularly reflecting glass material. The polarimetric bi-directional reflectance distribution function (pBRDF) describing the paint and metal surfaces are based on RIT measurements previously reported upon [4], while the rubber material $\mathrm{pBRDF}$ is derived from parameters for a similar material in the Nonconventional Exploitation Factors (NEF) database, and the glass pBRDF based on first principles knowledge of glass and it's complex index of refraction in the LWIR region of the spectrum. The plot in Figure 1 shows Stoke's $\mathrm{S}_{0}$ and $\mathrm{S}_{1}$ components of the modeled polarized emissivity vector $\left[\varepsilon_{0}, \varepsilon_{1}, \varepsilon_{2}, \varepsilon_{3}\right]$, where $\mathrm{e} 2$ and e 3 are assumed to be zero based on first principles knowledge of the emission polarization physics and chosen Stoke's coordinate system.

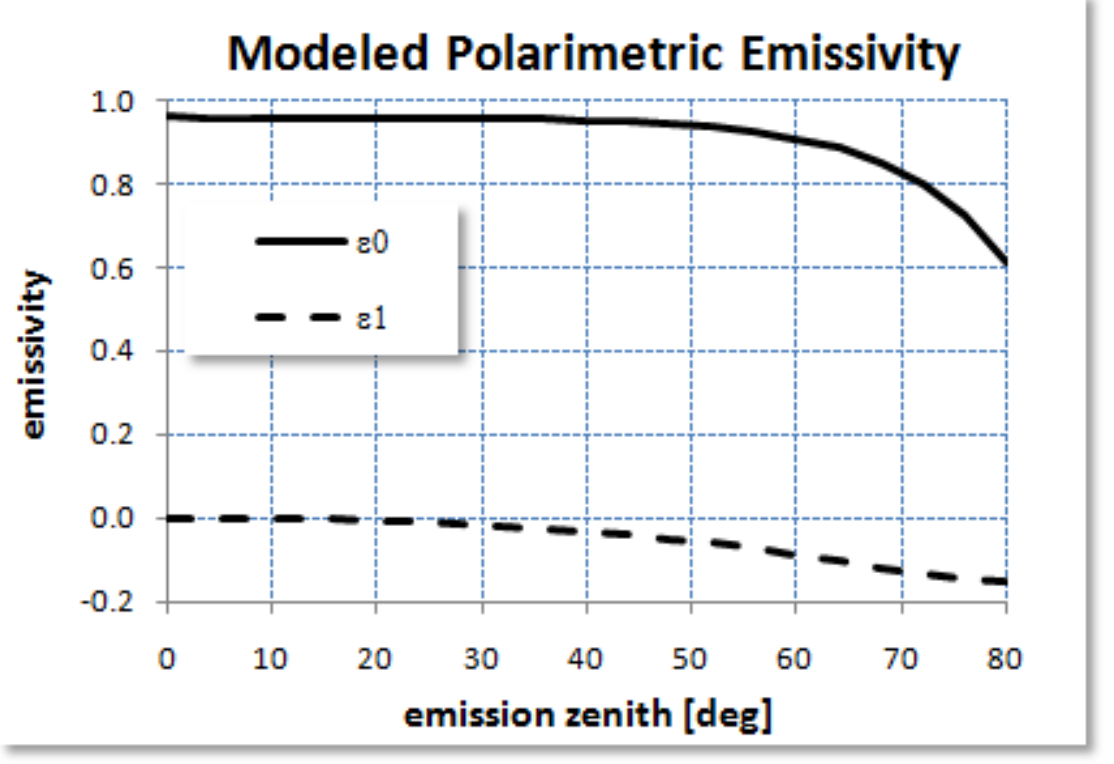

Figure 1. Plot of modeled polarimetric emissivity for car paint surface, derived from fit with measured data.

The automobile geometries are based on Google 3D warehouse geometries available for public use [3]. We chose a Chevy 454, Mazda Miata and Volkswagon Beetle for this study due to their unique geometric characteristics (see Figure 2).
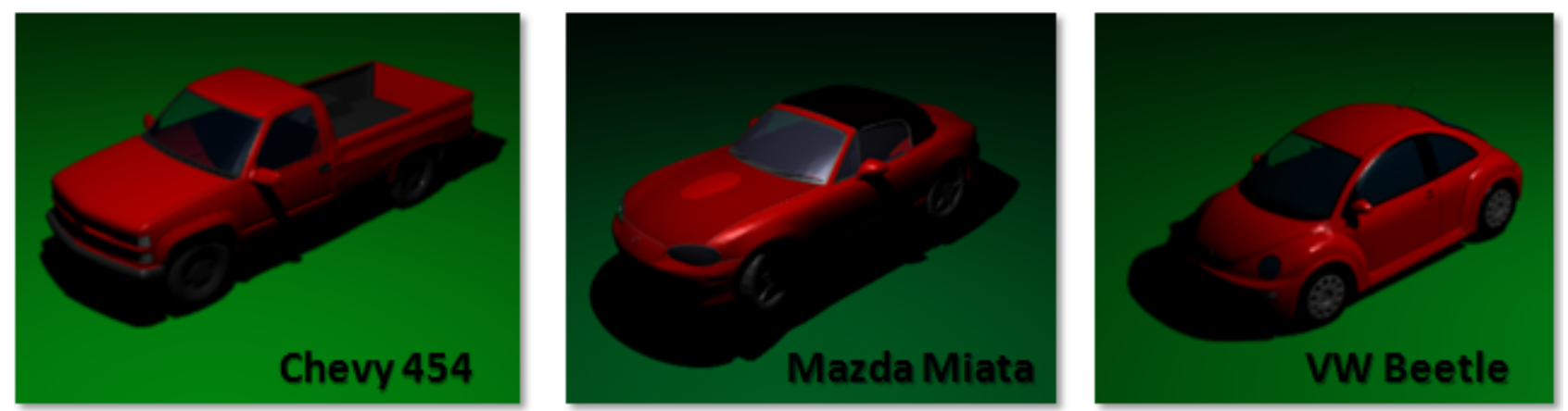

Figure 2. RGB renderings of three target vehicles utilized for this study. 
The imaging platform to be modeled is a broadband long-wave infrared (LWIR) polarimetric with a flat spectral response function. No noise or MTF effects are modeling in this preliminary phase, therefore all simulated imagery represents aperture reaching radiance (in $\mathrm{W} / \mathrm{cm}^{2} / \mathrm{sr}$ ). Additionally, we assume the background and skydome (or lack thereof) is well characterized. The altitude of the imaging platform is held constant, while the platform is allowed to fly over the target for a range of imaging sensor elevation angles (ISEL) from 0 to 90 degrees. However, the lower ISEL imagery will have a significant increase in ground sample distance relative to nadir imagery, which is accounted for in the simulation process.

\subsection{Target Signature Space Generation}

The target signature space we are interested in generating how the automobiles would appear as if they were unresolved by the LWIR polarimetry (target is fully contained within a single pixel, ignoring optical and other point spread function effects for now). To accomplish the single pixel response that would originate from imaging the vehicles, we utilize DIRSIG to render a $128 \times 128$ spatially oversampled image for 12 different zenith angles ranging from 0 to 82.5 degrees and 60 different azimuth angles ranging from 0 to 334 degrees for a total of 720 different target views. The resulting hemispherical target space may be linearly interpolated to a $128 \times 128$ polar plot image (Figure 1 for example) for visualization. The gray level of the polar images represents a fundamental radiometric quantity such as a single component of the pixel Stoke's vector, or a derived radiometric quantity such as degree of linear polarization (DOLP) or angle of polarization (AOP). The center of the hemispherical target spaces correspond to a nadir view geometry, while increasing away from the center represents an increase in zenith angle away from nadir and the azimuth angle with the space corresponds to the absolute azimuth angle between the automobile and the imaging platform.

The notional sensing platform is expected to maintain a constant altitude as it passes over the target, acquiring multiple images of the target from multiple view angles. The example in Figure 3 shows two different potential platform trajectories across the hemispherical space relative to the target where the imaging opportunities are represented by the star graphics.

\subsection{Target Pose Estimation}

In order to estimate the target pose, we wish to find the best match between the background removed target signature $S_{\mathrm{tgt}}(\theta, \phi)$

$$
S_{\text {tgt }}(\theta, \phi)=\left[\begin{array}{l}
S 0_{\text {tgt }} \\
S 1_{\text {tgt }} \\
S 2_{\text {tgt }}
\end{array}\right]=\left[\begin{array}{l}
S 0_{\text {pixel }} \\
S 1_{\text {pixel }} \\
S 2_{\text {pixel }}
\end{array}\right]-\left[\begin{array}{l}
S 0_{\text {bg }} \\
S 1_{\text {bg }} \\
S 2_{\text {bg }}
\end{array}\right]
$$

and the measured background removed Stoke's vector. Additionally, the matching between modeled signatures and measured signatures may also take place against the derived quantities degree of linear polarization (DOLP) and angle of polarization (AOP). The resulting azimuthal orientation of the platform pass through the modeled data is then utilized to estimate the azimuthal orientation or pose of the target vehicle. The actual fitting process may utilize a non-linear least squares approach that minimizes the cost function:

$$
\mathrm{E}(\gamma)=\frac{1}{2 \pi} \sum_{i=1}^{N}\left(A O P_{\text {meas }, i}-A O P_{\text {model }}\left(\theta_{i}, \gamma+\phi_{i}\right)\right)^{2}+\sum_{i=1}^{N}\left(D O L P_{\text {meas }, i}-D O L P_{\text {model }}\left(\theta_{i}, \gamma+\phi_{i}\right)\right)^{2}
$$

where the parameter $\gamma$ is the relative azimuth between the modeled target space and the actual target space permitting estimation of vehicle pose. 


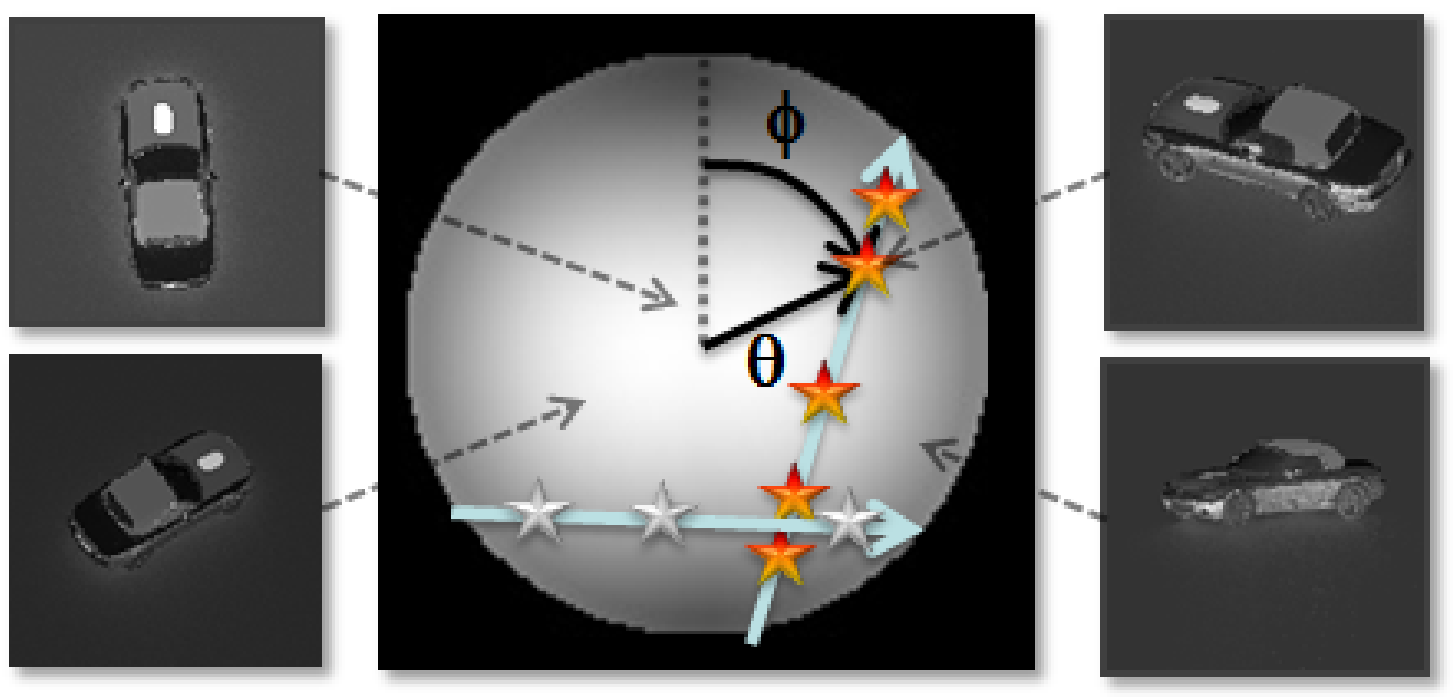

Figure 3. Example of an un-resolved hemispherical target space for the $\mathrm{S}_{0}$ component of a Mazda Miata on a warm uniform background. The two arrows represent two potential passes through the target space by a notional platform.

\subsection{Experimentally Captured LWIR Stokes Imagery}

For an initial evaluation of the modeled target space, we acquired LWIR linearly polarized intensity bands at angles of 0 , 45,90 , and 135 to the horizontal plane permitting derivation of the Stoke's S0, S1, and S2 images of a vehicle on a warm background. The imagery was acquired with the Corvus Long-Wave polarimeter having performance and design specifications listed in Table 1 below.

The vehicle target utilized for the collections was a Mazda Miata with a soft top deployed. The acquisition scenario consisted of imaging the target from a nearby rooftop, moving the vehicle relative to it's background between each series of intensity images. Relative target azimuth values of $0,66,114,150$, and 180 degrees were collected with an imaging zenith of approximately 61 degrees from nadir. Additionally, the background environment of target vehicle was quite complex, consisting of dozens of adjancent cars and buildings (not visible directly in the imagery, but some visible in the polarized reflections from the horizontal car surfaces). The Stokes imagery in Figure 4 below shows the vehicle in a position having a relative azimuth of 66 degrees to the camera line of sight. 


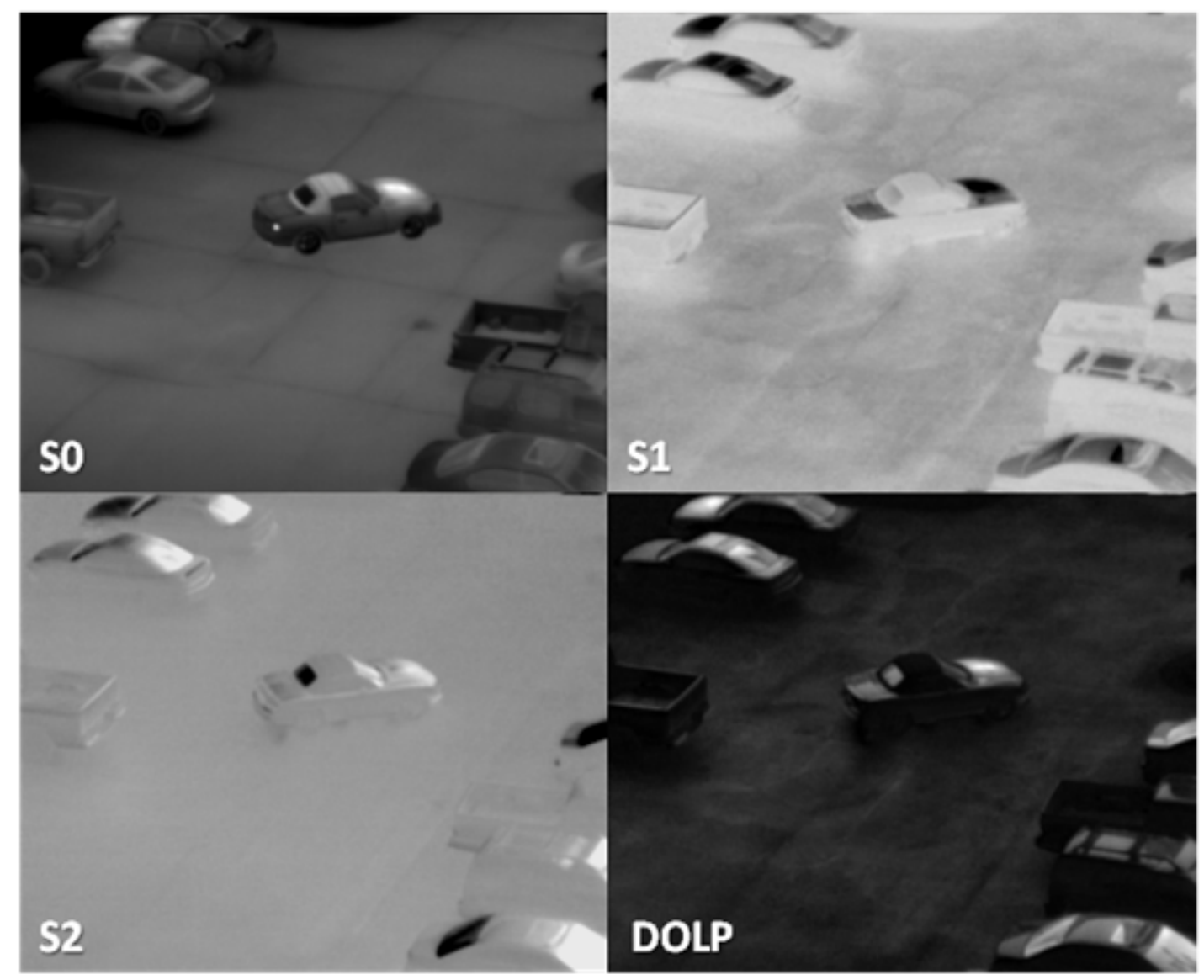

Figure 4. Sequence of S0, S1, S2, and derived DOLP image of vehicle target in cluttered urban background.

\begin{tabular}{r|c|c|} 
Property & Specification & Units \\
\hline Bandpass & $7.5-13.5$ & microns \\
\hline FPA format & $320 \times 240$ & pixels \\
\hline FPA type & microbolometer & - \\
\hline Pixel pitch & 38 & microns \\
\hline Field of View & $10 \times 13$ & degrees \\
\hline Polarization & linear & - \\
\hline Stokes Precision & 0.25 & $\%$ \\
\hline Polarimeter type & Rotating retarder & - \\
\hline Frame rate & 30 & $\mathrm{~Hz}$ \\
\hline Interface & GigE & - \\
\hline Size & $10 \times 6 \times 7.5$ & inches \\
\hline Weight & 12 & lbs \\
\hline
\end{tabular}

Table 1. Corvus Long-Wave polarimeter performance and design specifications. 


\section{RESULTS}

Our results are a mixing of modeling target space predictions and best effort comparison to experimentally acquired target signatures. First we examined a series of simple geometric shapes on a cold, non-reflecting background. Next we generated target signature predictions for the three different vehicle shapes on a cold, non-reflecting background. Finally, we picked the Mazda Miata target geometry and examined the effect of introducing a warm background, with some polarized reflection properties (about 3\% across the LWIR) representative of concrete or asphalt.

\subsection{Modeled Target Space for Simple Object Shapes}

In order to better understand what the target signature space represents, we generated the S0, S1, S2, DOLP, and AOP signatures for a simple rectilinear box, a flat ground plate, and a spherical surface (see Figure 5). As expected, the spherical surface S0 signature is independent of view angle, resulting in a uniform tone in the graphic in Figure 5. Additionally, within some quantization noise due to the finite nature of the spatial oversampling, the net S1 and S2 signatures were very close to zero as expected. Additionally, although the AOP and DOLP target spaces for the sphere show some structure, this structure is basically meaningless since the magnitude of the polarization is on the order of $10^{-5}$ and due only to sampling artifacts in the simulations.

Next, the ground plate target shows a falloff in S0 signature with increasing view zenith, as expected. Additionally, all of the polarization signature is in the S1 band which we expect based on the orientation of the camera system and the fact that the plate is flat to the ground (in the 'horizontal' plane we define for horizontal polarization).

Lastly, we examined the target space signatures for a rectilinear box. The S0 signature has a maximum when the box is oriented with its long side facing the camera system as expected. Additionally, there is also the expected symmetry about a 180 degree rotation in all other signatures which follows from the rotational symmetry of the target itself. In the case of this simple rectilinear box, we cannot discriminate between the front and back of the box, but we can discriminate between the front and a side of the box.

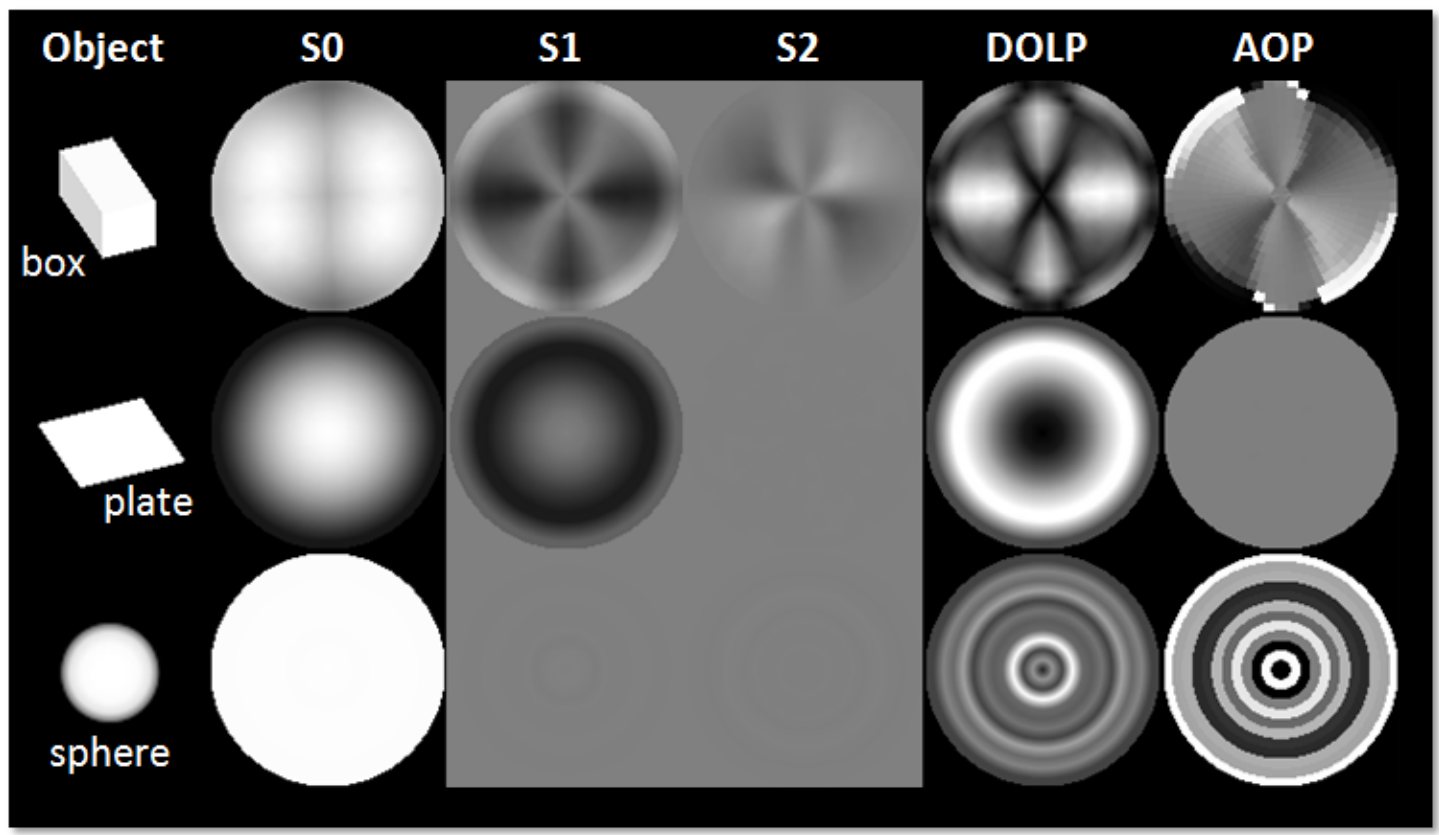

Figure 5. S0, S1, S2, DOLP and AOP target space visualizations for simple spherical, rectilinear box, and flat plate objects. 


\subsection{Modeled Target Space for Vehicles}

Next, we generated similar target space representations (Figure 6) for a pickup truck, a Miata, and a VW Beetle. Although there does not appear to be a significant contract between the three vehicles in the S0 signature, there is a definite contrast and uniqueness to the target space for each of other polarimetric bands (S1, S2, DOLP and AOP). This takeaway is key, in that it gives weight to the idea that we may be able to utilize polarimetry to discriminate not only the pose of an object, but also its shape.

Although the S1 and S2 target spaces are different for each object, the best discrimination (based on visual examination only) appears to be in the derived polarimetric quantities DOLP and AOP. More specifically, the minimum values of DOLP for each object lie in different regions of the target space. Although it is not feasible to expect an operational polarimetric camera to acquire signatures across the full $2 \pi$ steradian space, the path the imager traverses through the target space may be move through a region that may best discriminate the pose and shape of an object of interest.

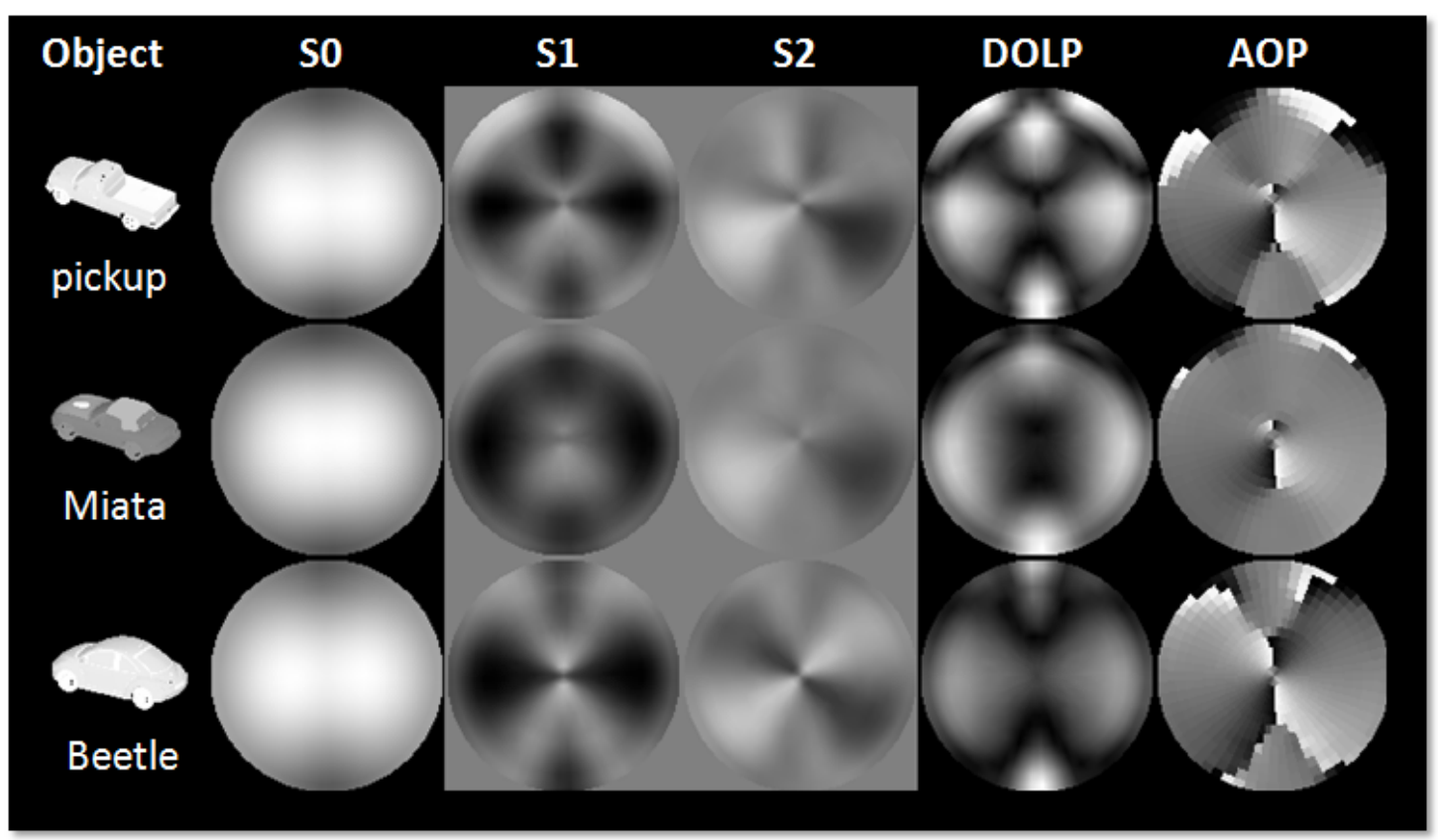

Figure 6. S0, S1, S2, DOLP and AOP target space visualizations for three different vehicle shapes.

Finally, we chose the Mazda Miata target and placed it on a warm background modeled to have the polarimetric emissive and reflective properties of a concrete material. We find the raw signature of the Miata on this background to appear quite different than the signature predicted for a cold, non-reflecting background. In fact there are multiple reasons for this. First off, the background concrete material imparts a significant signature on its own that resembles the flat plate signature to some extent. However, due to multiple bounce effects (ie. thermally emitted ground radiance reflecting from the side of the car) the resulting signature is not simply a weighted linear combination of the Miata only and the background only signatures. In fact the multi-bounce effects are quite significant and have a strong influence on the net signatures of the unresolved target and must be taken into consideration.

However, to increase the contrast of the target spaces for visualization, we remove the signatures we'd expect from the concrete alone resulting in a 'background-removed' target space (see the middle row of Figure 7). This background removal step helps to restore the signature of the Miata on the warm background to something that better resembles the signature of the Miata on a cold, non-reflecting background. However the restoration is not possible in full due to multibounce effects. In fact the multi-bounce of the ground thermally emitted radiance from the car side actually dominates 
the S1 signature and flips the sign of it in the azimuthal orientations where the side of the car is facing the camera (90 and 270, or East and West on the hemispherical target space visualization).

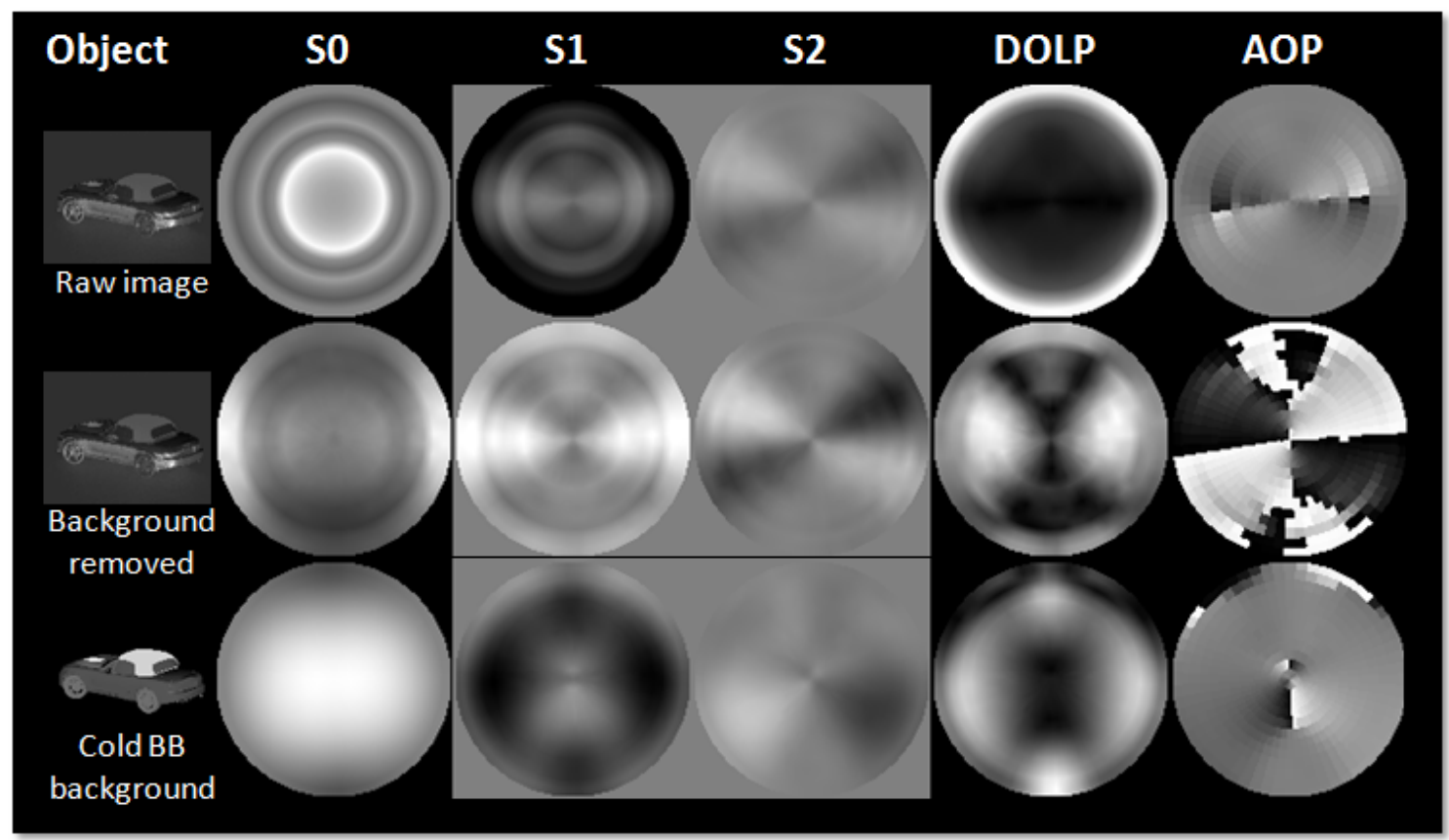

Figure 7. S0, S1, S2, DOLP and AOP target space visualizations for a Mazda Miata on a warm background, one a warm background with the background bias removed, and on a cold blackbody background for comparison.

\subsection{Comparison of Modeled and Measured Target Spaces}

Finally, we down-sampled the imagery of the Mazda Miata acquired with the Corvus LWIR polarimeter to represent single pixel signatures for the 5 vehicle azimuths. For the verification of the modeled target signatures we plot an azimuthal slice, taken at the camera zenith of around 61 degrees from nadir, of the target space as a curve and the measured signatures as the red diamond points (see Figure 8 below).
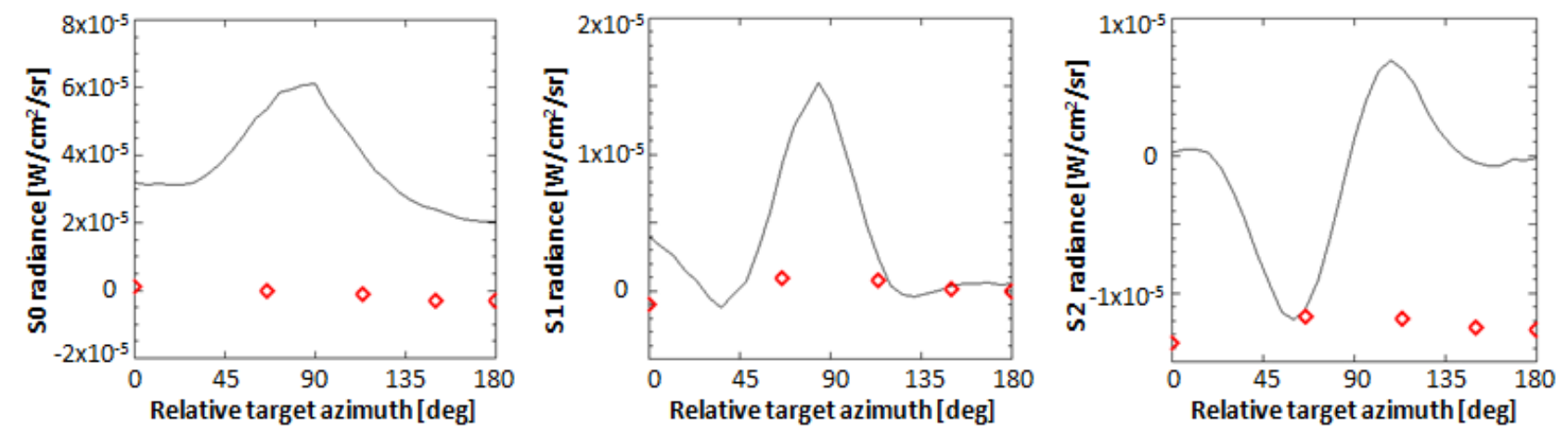

Figure 8. A comparison of the measured (diamonds) and modeled (curve) target signatures for a Mazda Miata on a warm background. 
As rigorous as the radiometry in the modeling was, the agreement between the modeled and measured S0, S1, and S2 signatures for the 5 vehicle azimuths is quite poor. Close examination of the measured Corvus imagery indicates a significant effect due to radiance originating from background objects being reflected from the Miata surfaces. A few notable examples of this phenomenology existing in the measured data (that does not exist in the modeled data) is the reflection of buildings outside of the field of view of the camera from the vehicle surface. More specifically, the reflection of a warm building from the Miata trunk is clearly visible in the DOLP image in Figure 4. Another example is the reflection of adjacent objects from the back bumper of the car in Figure 4. This bright reflection actually negates the expected positive (emitted S1 is negative for horizontal surface and positive for vertical surface) S1 signature, whereas the modeled signature has a strongly positive S1 signature on the back bumper due to emission and less background interaction.

Although the modeled and measured target signatures show poor agreement, close inspection of the details missing from the modeled scene (buildings, adjacent cars etc.) explain this result. From a pure visual perspective (see Figure 9 below), we are confident in the accuracy of the radiometry in the modeling software, but realize that verification of the proposed pose estimation approach will require either a re-collection of measured imagery under less cluttered background conditions or inclusion of all of the background objects in the modeled scene.
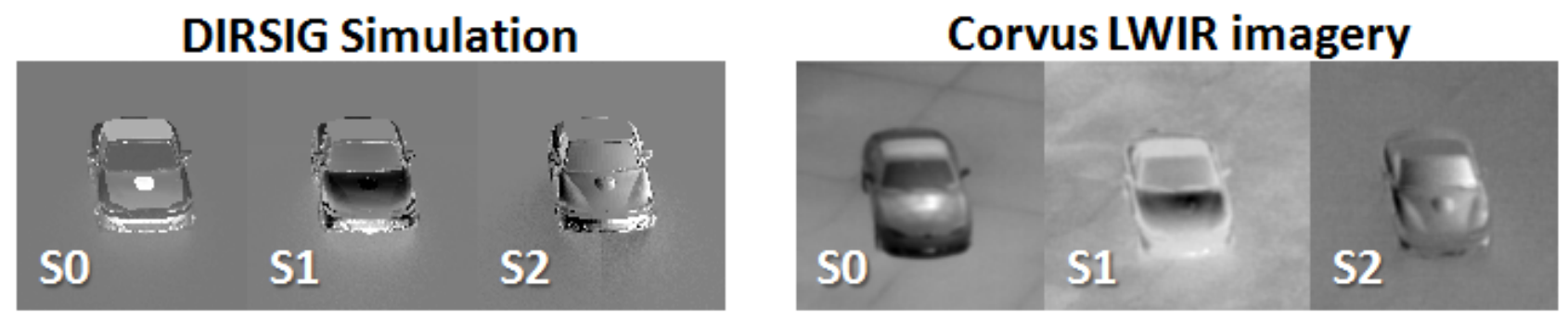

Figure 9. A comparison of modeled (left) and measured (right) Stokes band images of a Mazda Miata on a warm background shows good qualitative agreement.

\section{CONCLUSION / FUTURE DIRECTIONS}

We have presented an approach for utilizing first-principles, physics-based modeling to predict the polarimetric signature of an unresolved object. Although the un-polarized S0 signature does not always permit good discrimination of target pose and shape, the addition of the polarimetric bands S1 and S2 and other derived polarimetric quantities expands the target space that we believe will indeed aid in discriminating the shape and pose of unresolved objects. More specifically, we proposed the idea of generating a two-dimensional, polarimetric target space representing the full $2 \pi$ steradians of possible camera viewing angles. A non-linear least squares fitting approach could then be utilized to match real measurements of unresolved objects within the modeled target space of one or more candidate targets to estimate target pose and potentially shape.

Within the context of this research, we were not able to find a strong quantitative agreement between the modeled target signatures and the handful of measured signatures due to details present in the real cluttered urban scene not captured in the modeled target space (such as adjacent buildings and vehicles). However, we anticipate future work will address this shortcoming by improving ground truth collection of adjacent objects in the measured imagery and a fuller representation of the true background environment of the target in the modeling environment. It is possible that the effort required to accurately model a very complex urban environment for accurate target space generation is not worth the effort, limiting the scope and applicability of our pose estimation approach to less cluttered scenarios. 


\section{ACKNOWLEDGEMENTS}

Michael Gartley would like to acknowledgement the support of the Intelligence Community Post-doctoral Research program.

\section{REFERENCES}

[1] J. S. Tyo, D. L. Goldstein, D. B. Chenault, and J. A. Shaw, "Review of passive imaging polarimetry for remote sensing applications," Appl. Opt., vol. 45(22), pp. 5453-5469, Aug. 2006.

[2] F. Goudail, P. Terrier, Y. Takakura, L. Bigu, F. Galland, and V. DeVlaminck, "Target detection with a liquid crystal-based passive stokes polarimeter," Appl. Opt., vol. 43(2), pp. 274-282, Jan. 2004

[3] M. Gibney, T. Rogne, and R. Tippets, "Shape Information from Polarimetric Measurement", IRIS Passive Sensors Conference, March 2000.

[4] M. Gartley, S. Brown, J. Schott, N. Sanders, A. Goodenough, "Polarimetric Scene Modeling in the Thermal Infrared", Proceedings of the SPIE, Polarization Science and Remote Sensing III, v6682-11, August 2007. 